\title{
LC determination of loratadine and related impurities
}

\author{
F.J. Rupérez, H. Fernández, C. Barbas* \\ Facultad de CC Experimentales y de la Salud, Universidad San Pablo-CEU Urbanización Montepríncipe, \\ Ctra. Boadilla del Monte, km 5,3-28668 Madrid, Spain
}

Received 31 October 2001; received in revised form 7 December 2001; accepted 16 December 2001

\begin{abstract}
Loratadine, an antihistamine, could include in its raw material seven impurities that ought to be separated identified and quantified for drug development and quality control. A HPLC method employing a SymmetryShield RP8 column has been developed and validated for loratadine and related compounds measurement, the last ones under the $0.1 \%$ level. The mobile phase consisted of methanol-buffer A (65:35, v/v), being buffer $\mathrm{A}: \mathrm{H}_{3} \mathrm{PO}_{4} 10 \mathrm{mM}$ $\left(\mathrm{H}_{2} \mathrm{O}\right)$ brought up to $\mathrm{pH} 7.00$ with triethylamine. UV detection was performed at $244 \mathrm{~nm}$. Validation parameters for linearity, accuracy and precision are in agreement with ICH guidelines for all the analytes and that permits to consider the method reliable and suitable for application to long-term stability and purity studies. (C) 2002 Elsevier Science B.V. All rights reserved.
\end{abstract}

Keywords: Loratadine; Impurities; HPLC

\section{Introduction}

Loratadine is a long-acting tricyclic antihistamine with selective peripheral histamine H1-receptor antagonistic activity. Loratadine is a white powder not soluble in water, but very soluble in organic solvents. Its chemical name is ethyl 4-(8chloro-5,6-dihydro-11 H-benzo [5,6] cyclohepta [1,2-b] pyridin-11-ylidene)-1-piperidine carboxylate. It is possible that bulk loratadine could include seven impurities that ought to be separated, identified and quantified in its analysis. Its formula and those corresponding to its impurities can be seen in the Fig. 1, where it can be observed

* Corresponding author. Fax: + 34-91-3510475.

E-mail address: cbarbas@ceu.es (C. Barbas). that the main component and structurally-related impurities posses in most of them similar structures and thus physico-chemical properties, which make resolution difficult.

Impurity profiling is an important issue in pharmaceutical analysis, particularly during product development and quality control.

Few analytical methods have been described for loratadine, most of them have been developed for pharmacokinetic studies and they are applied to quantify loratadine and its metabolite descarboethoxyloratadine (LD) in plasma by HPLC [1], GC-MS [2], GC with N-P detector [3]. Three methods have been described for loratadine assay in pharmaceutical preparations: a polarographic method [4] which requires previous derivatization, a spectrophotometric and a HPLC methods [5], 
but none of them permit impurity evaluation. The determinations of drug-related impurities are generally performed by HPLC, which is an established technique with highly automated instrumentation. The standard requirements of such an impurity method are [1] that all likely synthetic and degradative impurities are resolved from each other and the main drug, and [2] that impurities can be monitored at the $0.1 \%$ level or below.

The present paper is dedicated to the development and validation of a HPLC method for the identification and quantification of loratadine and its related impurities in raw material and in tablets as pharmaceutical presentation.

\section{Experimental}

\subsection{Apparatus}

A Beckman (Palo Alto, USA) HPLC system provided with a 116 pump, an automatic injec-

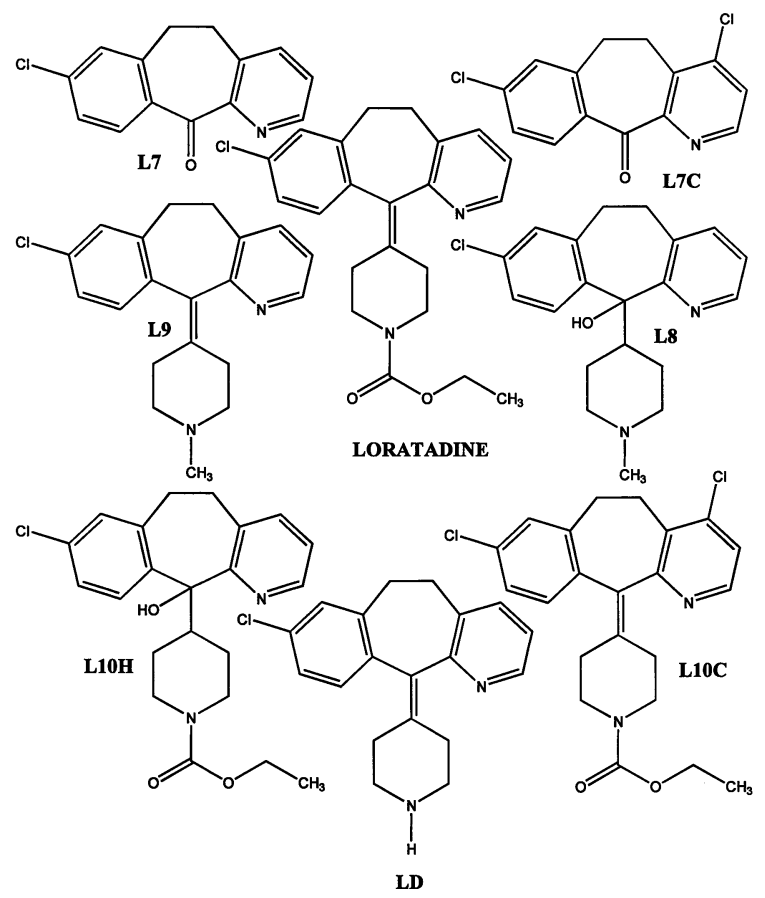

Fig. 1. Chemical structures of loratadine and related impurities. tor (507e), a 166 UV detector and a Gold System data processor were used. The chromatographic analysis were performed on a 5 $\mu \mathrm{m}$ particle SymmetryShield RP8 (Waters, Madrid, Spain) column $(25 \times 0.46 \mathrm{~cm})$ kept in a Thermoquest (Madrid, Spain) Gecko 2000 column oven at $40{ }^{\circ} \mathrm{C}$. Different chromatographic columns with the same size $(25 \times 0.46$ $\mathrm{cm})$ were also assayed: SymmetryShield ${ }^{\circledR} \mathrm{RP}_{18} 5$ $\mu \mathrm{m}$ (Waters, Madrid, Spain), Phenomenex Synergi $^{\circledR}$ MAX-RP $4 \mu \mathrm{m}$ (Micron Analítica, Madrid, Spain), Phenomenex Luna ${ }^{\circledR}$ C8 $5 \mu \mathrm{m}$ (Micron Analítica, Madrid, Spain), and Tracer Kromasil C8 $5 \mu \mathrm{m}$ (Teknokroma, Madrid, Spain), and results were compared.

Final mobile phase consisted of methanolbuffer $\mathrm{A}(65: 35, \mathrm{v} / \mathrm{v})$, being buffer $\mathrm{A}: \mathrm{H}_{3} \mathrm{PO}_{4} 10$ $\mathrm{mM}\left(\mathrm{H}_{2} \mathrm{O}\right)$ brought up $\mathrm{pH} 7.00$ with triethylamine. The flow rate was $1 \mathrm{ml} / \mathrm{min}$ and the injection volume was $20 \mu \mathrm{l}$. UV detection was performed at $244 \mathrm{~nm}$ and peaks were identificated with retention times as compared with standards.

\subsection{Chemicals}

Standards of loratadine and impurities as well as tablets and placebo of the speciality were kindly provided by CINFA, S.A. (Pamplona, Spain). Phosphoric acid $85 \%$ and triethylamine were from Merck (Darmstadt, Germany), and other organic solvents from Scharlau (Barcelona, Spain).

\subsection{Optimisation of HPLC method}

Selectivity under RP-HPLC conditions can be controlled by mobile phase composition, $\mathrm{pH}$, temperature and stationary phase chemistry.

On the other hand, the different performance of chromatographic columns with silica bonded to $\mathrm{C} 8, \mathrm{C} 12$ or $\mathrm{C} 18$ and, in some cases, coming from different manufacturers and with different groups employed for the bounding of the hydrocarbonated chain to the silica have been compared in relation to loratadine and its impurities separation. 


\subsection{Standard solutions and sample preparation}

Loratadine stock solution was prepared with $156 \mathrm{mg}$ of loratadine exactly weighed and dissolved in a $100 \mathrm{ml}$ volumetric flask with methanol. Stock solutions of every impurity were individually prepared with $10 \mathrm{mg}$ exactly weighed and dissolved in $10 \mathrm{ml}$ volumetric flasks with methanol. An intermediate solution containing all the impurities was prepared with $1 \mathrm{ml}$ of each impurity stock solution, all together, brought up to $10 \mathrm{ml}$ with methanol.

For quantitation $67 \mathrm{mg}$ of the pulverised tablets were made up to $25 \mathrm{ml}$ with mobile phase. After water bath sonication for around 10 min samples were filtered with $0.45 \mu \mathrm{m}$ nylon filters prior to the injection. The standard was prepared with 4 $\mathrm{ml}$ of stock solution of loratadine made up to 25 $\mathrm{ml}$ with mobile phase.

\subsection{Validation}

The linearity study verifies that the sample solutions are in a concentration range where analyte response is linearly proportional to concentration. For main component assay methods, this study is generally performed by preparing standard solutions at five concentration levels, from 50 to $150 \%$ of the target analyte concentration. In this case loratadine concentrations were from 125 to 374 $\mu \mathrm{g} / \mathrm{ml}$. They were prepared in $25 \mathrm{ml}$ volumetric flasks with 2-6 $\mathrm{ml}$ of stock loratadine solution plus methanol to complete $6 \mathrm{ml}$ and mobile phase to make the volumes. Each point was analysed three times. For sample linearity five solutions were identically prepared, but with the proportion of the excipients of the speciality $(61 \mathrm{mg})$ added to each flask.

For impurity methods, linearity is determined by preparing standard solutions at five concentration levels over a range such as $0.05-0.25 \mathrm{wt} . \%$. In this case standards ranged from 0.1 to 0.5 $\mu \mathrm{g} / \mathrm{ml}$ and they were prepared in $25 \mathrm{ml}$ volumetric flasks containing $4 \mathrm{ml}$ of loratadine stock solution with $30,45,62,94,110$ and $125 \mu$ of the intermediate impurities solution and they were levelled off with mobile phase. Six points were prepared instead of five just in case there were sensitivity problems in the lower value. Samples linearity was tested in the same way but with the proportional weight of the excipients of the speciality (61 $\mathrm{mg}$ ) added to each flask.

The accuracy of a method is the closeness of the measured value to the true value for the sample. For pharmaceutical studies, the most widely used approach is the recovery study, which is performed by spiking analyte in blank matrices. It was tested in the same linearity assay for both main component and impurities. The percent recovery and R.S.D.s were then calculated.

The precision of an analytical method is the amount of scatter in the results obtained from multiple analyses of an homogeneous sample. The first type is repeatability or intra-assay precision. Intra-assay precision data were obtained by repeatedly analysing, in one laboratory on day one, six aliquots of a homogeneous sample, each of which was independently prepared according to the method procedure. The second type is intermediate precision. These data were obtained by repeating the intra-assay experiment on a different day with newly prepared mobile phase and samples.

The detection limit of a method is the lowest analyte concentration that produces a response detectable above the noise level of the system, typically, three times the noise level. The detection limit needs to be determined only for impurity methods in which chromatographic peaks near the detection limit will be observed.

The quantitation limit is the lowest level of analyte that can be accurately and precisely measured. This limit is required only for impurity methods and the best option is to have it determined by reducing the analyte concentration until a level is reached where the precision of the method is unacceptable. As a theoretical approach, that ought to be checked, the quantitation limit is often calculated as the analyte concentration that gives $S / N=10$.

Standards stability was tested by running the same sample corresponding to the medium point in the linearity assay for $0-3$ and 7 days and with the same mobile phase. Between runs, solutions were stored at $4{ }^{\circ} \mathrm{C}$. The initial area was considered $100 \%$ and recoveries in the following days were evaluated. 


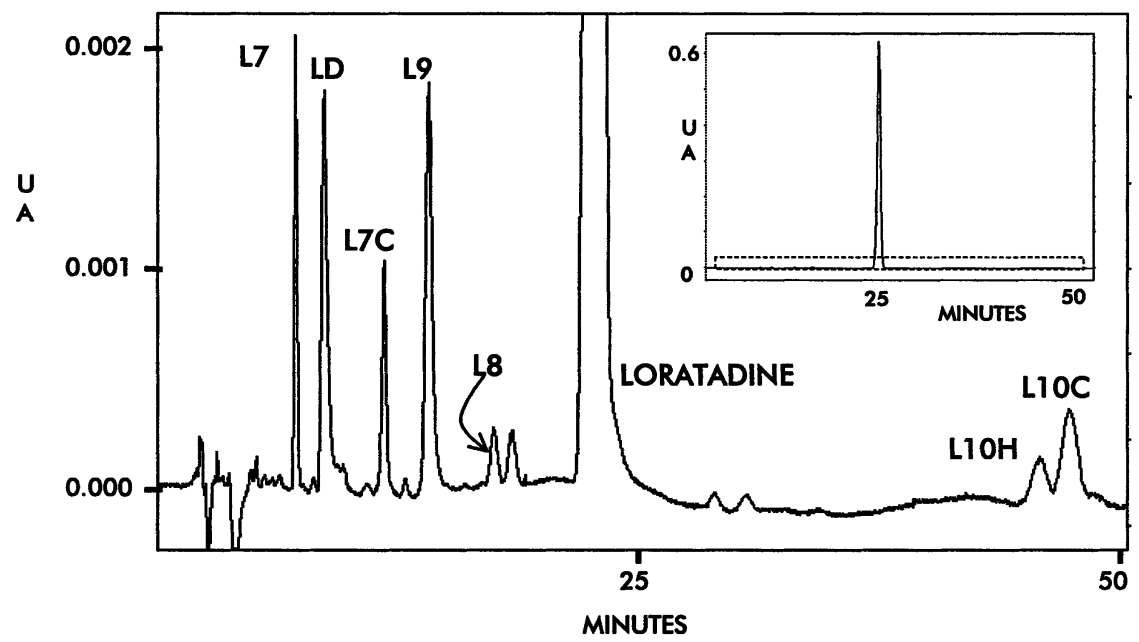

Fig. 2. Chromatogram showing loratadine $(0.2 \mu \mathrm{g} / \mathrm{ml})$ and related impurities (all of them $0.1 \%$ of the main peak) separation. The mobile phase consisted of methanol-buffer $\mathrm{A}(65: 35, \mathrm{v} / \mathrm{v})$, being buffer $\mathrm{A}, \mathrm{H}_{3} \mathrm{PO}_{4} 10 \mathrm{mM}\left(\mathrm{H}_{2} \mathrm{O}\right)$ brought up to pH 7.00 with triethylamine. Flow rate, $1 \mathrm{ml} / \mathrm{min}$ and UV detection, $244 \mathrm{~nm}$.

\section{Results and discussion}

In previous times many HPLC methods suffered from problems when analysing basic drugs, such as loratadine, since these compounds strongly interact with polar ends of HPLC column packing materials, causing severe peak asymmetry and low separation efficiencies. High purity silica backbone and advances in bonding technology have alleviated the tailing problem of polar compounds in HPLC to a significant extent.

Consequently, for the initial development a SymmetryShield RP8 column was used. This packing was selected because it has one of the lowest hydrophobicity and silanol activity as seen in commercial catalogues.

The $\mathrm{pH}$ value of 7.00 , in the mobile phase permitted a low ionization degree and therefore, a higher retention of the analytes. It provides more tools to obtain the separation. Moreover, it permits us to take advantage of the addition of a 'silanol blocker', such as triethylamine, to the mobile phase and this has proved to be necessary to obtain good peak symmetry in the present work.

The critical point in developing the separation was to get a good resolution for structurally similar compounds while eluting simultaneously im- purities of very different polarity. Gradient elution was avoided because, in our experience, it is troubleshooting when trying to determine impurities, because when the organic phase increases many times, unidentified impurities retained in the system coming from water or different sources begin to elute and quantitation of impurities in drifting baselines is difficult. Different methanol/ aqueous buffer ratios were assayed to obtain the final conditions described above, by which we were able to obtain the chromatogram represented in Fig. 2, with the seven impurities and loratadine well resolved. The elution order is difficult to explain in the pairs of compounds, which differ only in a double bound versus a hydroxyl group, such as L9 and L8 or loratadine and $\mathrm{L} 10 \mathrm{H}$. Later elution of hydroxylated compounds could be related to a loss of polarity in the pyridine ring because of the formation of intra-molecular hydrogen bounds as can be observed in Fig. 1.

Fig. 3 presents the separation obtained with three $\mathrm{C} 8$ columns of different brands, one $\mathrm{C} 12$ and one $\mathrm{C} 18$. The same mobile phase and chromatographic conditions were employed in all cases. Packing materials characteristics, given by the manufacturers, are summarised in Table 1. 


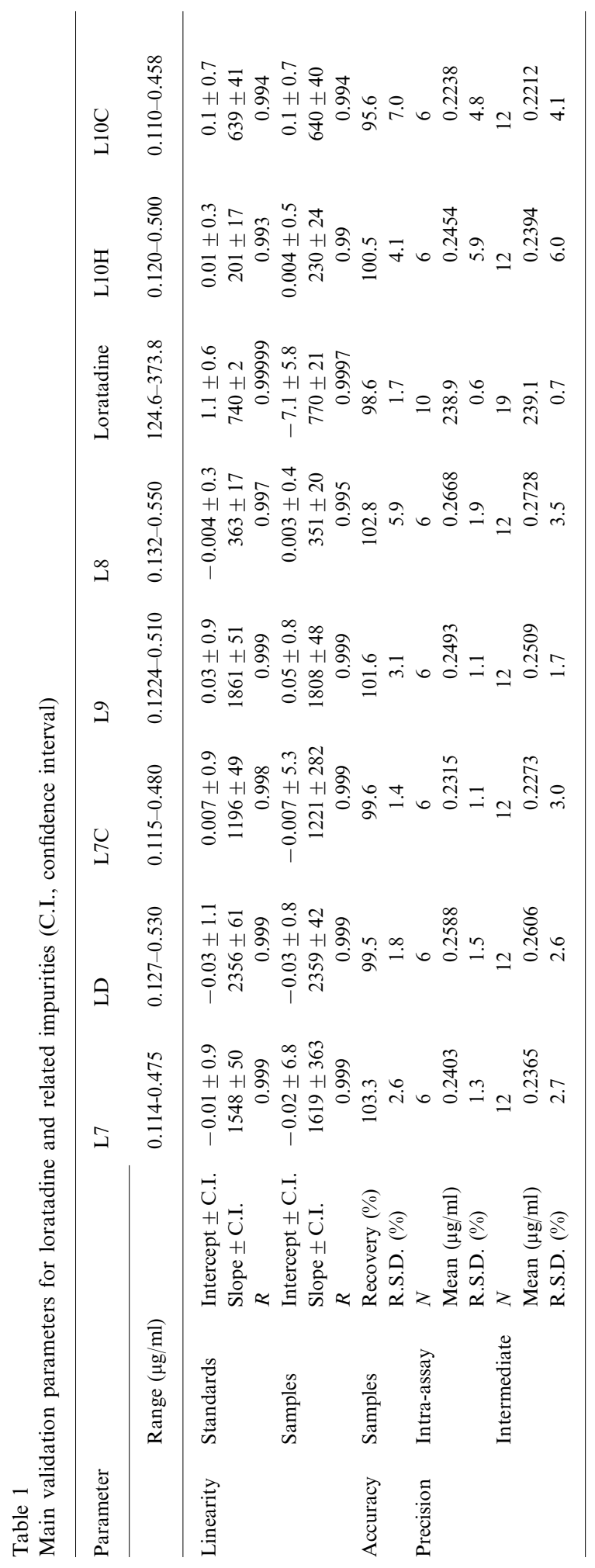


Synergi packing, in spite of being $\mathrm{C} 12$, presented higher retention times than SymmetryShield RP18. It could be explained with the pore size which is smaller in the Synergi than in the Symmetry and it provides a higher surface area (500 vs. $335 \mathrm{~m}^{2} / \mathrm{g}$ according to the manufacturers). It results in a phase with hydrophobic retention and methylene selectivity superior to Symmetry Shield RP18 column. On the other hand, Kromasil packing, the one described with higher silanol activity, changed the elution order of L8 and L9. Luna packing did not resolve LD and L7C and finally SymmetryShield RP18 and Symmetry Shield RP18 with low hydrophobicity are the packings which retain less to $\mathrm{L} 10 \mathrm{H}$ and L10C, the less polar compounds.

After development the method was validated for determining loratadine and related impurities in raw material and in tablets. The main validation parameters in both methods are shown in Table 2.

Both standards and samples show a good linearity for the eight analytes in the two methods with correlation coefficients over 0.999 for loratadine and over 0.99 for impurities and no appreciable bias was found. R.S.D. values were $<2 \%$ in the intra-precisions except for the two last compounds $\mathrm{L} 10 \mathrm{H}$ and $\mathrm{L} 10 \mathrm{C}$ due to their shorter peak heights. That causes a higher influence of baseline noise in their areas. R.S.D. values were $<4 \%$ in the intermediate precision except for $\mathrm{L} 10 \mathrm{H}$ and $\mathrm{L} 10 \mathrm{C}$ which are 6.0 and $4.1 \%$, respectively.

Recoveries do not statistically differ from $100 \%$ ( $t$-test, $P<0.05)$ in any case and R.S.D. for recoveries range from 1.4 to $7.0 \%$. Limits of quantification must be established with the lower concentrations in which the method can be validated with enough precision and accuracy. Experimental limits of quantification were established in $0.05 \%(0.1 \mu \mathrm{g} / \mathrm{ml})$ for the seven analytes. These limits are the lowest concentration values of the impurities measured in the validation and passing the acceptance criteria. Therefore, they are more reliable than the values obtained with mathematical aproaches, because all the impurities and the parent compound are present in the same run.

Calculated limits of quantification were determined for loratadine, just to compare with the experimental values. Two methods were employed: EURACHEM [6] for which six replicates of five points in the lower range $(0.05-0.25 \mathrm{wt} . \%)$ were measured. LOQ is established by representing concentration versus R.S.D. and interpolating

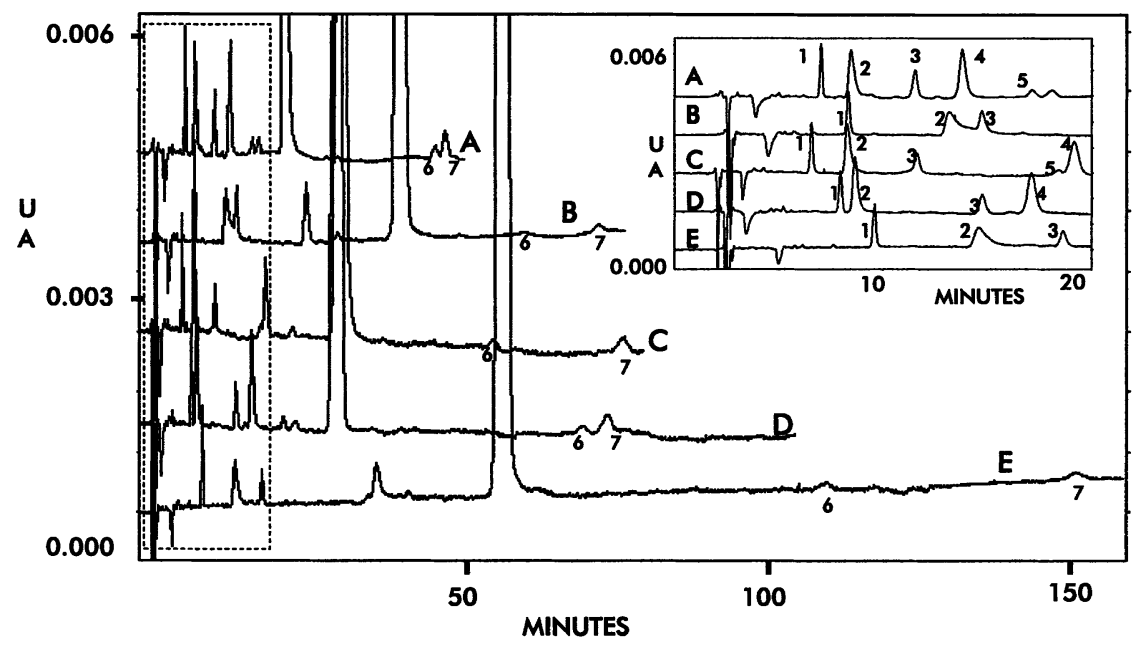

Fig. 3. Comparative chromatogramas obtained with different columns from top to bottom, (A) symmetryshield RP8; (B) Luna C8; (C) Kromasil C8; (D) symmetryshield RP18; (E) synergi RP 12. Peak identification, (1) L7; (2) LD; (3) L7C; (4) L9; 5, L8; (6) L10H; (7) L10C. Same conditions as in Fig. 2. Loratadine concentration is $0.2 \mu \mathrm{g} / \mathrm{ml}$ and related impurities are all of them $0.1 \%$ of the main peak. 
Table 2

Packing material characteristics

\begin{tabular}{llclcr}
\hline Packing material $25 \times 0.46 \mathrm{~cm}$ & Particle size $(\mu \mathrm{m})$ & Pore size $(\AA)$ & Pore volume $(\mathrm{ml} / \mathrm{g})$ & $\begin{array}{l}\text { Carbon load } \\
(\%)\end{array}$ & End capping \\
\hline Synergi C12 & & & & 15 & Yes \\
Kromasil C8 & 4 & 80 & 1.00 & 12 & Yes \\
Luna C8 & 5 & 100 & 0.9 & 14.75 & Yes \\
SymmetryShield RP8 & 5 & 100 & 0.9 & 15.0 & Yes \\
SymmetryShield RP18 & 5 & 100 & 0.9 & 17.0 & Yes
\end{tabular}

the concentration corresponding to $10 \%$ of R.S.D. and IUPAC method [7] which calculates the standard deviation of the blank by extrapolation to zero concentration a regression line in the lower range. The results obtained in HPLC were $0.19 \%$ by EURACHEM method, which is quite over the experimental value $(0.05 \%)$, and $0.06 \%$ by IUPAC method, which is nearest to the experimental value.

For stability of standards the recoveries after 7 days were $100.0 \%$ with a R.S.D. value of $0.6 \%$. Therefore, the standards can be considered stable for at least 1 week kept in solution at $4{ }^{\circ} \mathrm{C}$. On the other hand, during the validation, series of samples lasting for over $48 \mathrm{~h}$ were processed and the vials were kept in the injector tray at room temperature without changes in the signal.

Although a formal robustness assay has not been achieved, this method has been applied over 1 year for a stability test of a pharmaceutical formulation with assays at 0, 1, 3, 6, 9 and 12 months with different mobile phases and three different equipment has been employed and always the method has passed the system suitability test. Therefore, it can be considered robust.

\section{Conclusion}

A HPLC method has been developed for loratadine and related impurities measurement in raw material and tablets. Validation parameters permit the method to be considered adequate.

\section{Acknowledgements}

CINFA S.A. as promoter of the study.

\section{References}

[1] D. Zhong, H. Blume, Pharmazie 49 (1994) 736.

[2] J. Martens, J. Chromatogr. B 673 (1995) 183.

[3] R. Johnson, J. Christensen, C.-C. Lin, J. Chromatogr. B 657 (1994) 125.

[4] J.A. Squella, J.C. Sturm, M.A. Diaz, H. Pessoa, L.J. Nuñez-Vergara, Talanta 43 (1996) 2029.

[5] M. Nogowska, M. Zajac, I. Muszalska, Chem. Anal. (Warsaw) 45 (2000) 681.

[6] EURACHEM, Guidance Document, 1993, vol. Guide 25, No WGD 2.

[7] G.L. Long, J.D. Winefordner, Anal. Chem. 55 (1983) $712 \mathrm{~A}$. 